\title{
Besoins en eau des taurins et des zébus en zone tropicale (Afrique Occidentale Française)
}

\author{
Par J. PAGOT et R. DELAINE
}

\section{BUT DES RECHERCHES}

Lorsqu'on étudie en A.O.F. le comportement des taurins et des zébus entretenus en élevage extensif permanent par les éleveurs africains, on est frappé par la possibilité qu'ont ces animaux de survivre avec de très faibles rations d'eau.

Les essais entrepris au Centre de Recherches Zootechniques de l'A.O.F. avaient pour but de déterminer l'influence du climat sur les besoins en eau d'animaux entretenus en permanence sur un pâturage naturel d'une région à climat soudanien.

$\mathrm{Si}$, en zone tempérée, la régulation thermique se résume le plus souvent à une lutte contre le froid, en zone tropicale, les homéothermes ont à éliminer, en plus de la chaleur d'origine métabolique, celle reçue de l'ambiance et doivent, pour maintenir leur température constante, intensifier l'activité des mécanismes thermorégulateurs, les plus efficaces étant ceux qui déterminent une vaporisation d'eau : respiration, sudation, perspiration.

Ces mécanismes sont sous la dépendance directe des conditions climatiques. Ainsi, "dans la vaporisation pulmonaire, comme la température de l'air pulmonaire est constante et égale à la température corporelle ou très voisine d'elle, la quantité d'eau perdue dépend de l'humidité de l'air inspiré, de sa température, et du rythme de la ventilation pulmonaire. Dans le cas de l'élimination cutanée, l'importance de la perte

\footnotetext{
Les mayennes calculées sont accompagnées de leur erreur égale en valeur absolue à 1,96 fois l'écart type de la moyenne.

Les coefficients de corrélation sont suivis de deux nombres indiquant les limitos inférieure et supérieure de l'interval'e de confiance à $95 \%$ qui ont été calcu'ées par l'intermédiaire de la fonction $Z=$ arg. th. $r$.
}

d'eau par diffusion à travers les téguments dépend de la teneur en eau de l'organisme, de l'intensité de la circulation cutanée et de la perméabilité cutanée" (Simonnet, 7). Elle s'accroît quand la température s'élève et que le degré hygrométrique s'abaisse (Pinson, 5) (Whitehouse et coll. 8). La sudation est chez tous les bovins un facteur efficace de lutte contre la chaleur. (Brody, 1) (Seath-Miller, 6).

La couverture des besoins en eau sera assurée par l'eau bue, l'eau contenue dans les aliments solides et l'eau métabolique.

La quantité d'eau bue dans le cas d'abreuvement à satiété varie en fonction du régime alimentaire et en particulier avec la teneur en eau des aliments. Dans le cas d'élevage au pâturage en zone tropicale, il est obligatoire de tenir compte de cette teneur qui varie de 8 à 78 p. 100 suivant la saison. (Harker et coll., 9-10-1i).

\section{PROTOCOLE EXPERIMENTAL}

Les animaux, tous adultes, étaient divisés en deux lots; un lot de 6 zébus de la race peule du fleuve, à robe pie-noire, que l'on trouve au Soudan el en Haute-Volta, et un lot de 6 taurins de race n'dama, à robe unie fauve, qui se rencontre au Soudan, en Guinée, en Haute-Volta et en Côte d'Ivoire.

Le poids moyen des taurins a varié au cours des observations de 234 à $250 \mathrm{~kg}$, celui des zébus de 246 à $297 \mathrm{~kg}$.

Les animaux entretenus en permanence sur un pâturage naturel avaient la possibilité de s'abreuver à satiété chaque jour vers 15 heures.

La température ambiante et le degré hygrométrique étaient enregistrés à l'heure de l'abreuvement ; l'évaporation, au cours des 24 heures 
Tableau I

BESOINS EN EAU DES TAURINS ET DES ZÉEUS EN ZONE TROPICALE (A.0.F.)

\begin{tabular}{|c|c|c|c|c|c|c|}
\hline \multirow{2}{*}{ MOIS } & \multirow{2}{*}{$\begin{array}{l}\text { Température } \\
\text { moyenne } \\
\text { a } 45 \text { heures }\end{array}$} & \multirow{2}{*}{$\begin{array}{l}\text { Degré hygromé- } \\
\text { trique moyen } \\
\text { a } 15 \text { heures }\end{array}$} & \multirow{2}{*}{$\begin{array}{l}\text { Evaporation } \\
\text { journalière } \\
\text { moyenne }\end{array}$} & \multirow{2}{*}{$\begin{array}{l}\text { Teneur moy- } \\
\text { enne en eau } \\
\text { des fourrages }\end{array}$} & \multicolumn{2}{|c|}{ Eau consormée en litres } \\
\hline & & & & & ZÉBuS & TAURINS \\
\hline JANVIEM & 30,22 & 13,15 & 6,23 & 29,40 & 21,62 & 21,62 \\
\hline FEVRIER & 32,28 & 15,87 & 8,96 & 21,60 & 22,62 & 25,12 \\
\hline MARS & 32,94 & 13,75 & 9,48 & 9,56 & 23,74 & 22,74 \\
\hline AVRIL & 35,47 & 27,71 & 88,15 & 8,18 & 27,33 & 23,46 \\
\hline MAI & 38,71 & 28,71 & 8,11 & 10,09 & 28,85 & 23,28 \\
\hline JIN & 33,71 & 42,16 & 3,78 & 78,61 & 23,16 & 18,94 \\
\hline JUILLET & 30,18 & 59,20 & 2,54 & 75,25 & 19,66 & 16,08 \\
\hline AOUT & 28,42 & 65,25 & 1,51 & 74,07 & 11,33 & 7,55 \\
\hline SEPTEMBRE & 29,68 & 69,37 & 1,47 & 71,09 & 10,42 & 7,42 \\
\hline OCTOBRE & 32,05 & 41,30 & 2,66 & 62,83 & 12,19 & 13,00 \\
\hline NOVEMBRE & 31,15 & 42,16 & 3,35 & 51,87 & 12,49 & 16,16 \\
\hline DSCEMBRE & 28,25 & 27,37 & 6,85 & 41,86 & 16,49 & 19,74 \\
\hline
\end{tabular}

précédant l'abreuvement, était mesurée à l'aide d'un évaporomètre à rondelle de buvard.

Chaque semaine des échantillons de plantes fourragères les plus fréquentes étaient ramassés, le degré d'humidité était déterminé par pesée avant et après dessication à l'étuve à $100^{\circ}-$ $105^{\circ} \mathrm{C}$.

\section{BESOINS EN EAU}

Les consommations journalières moyennes furent minimales en Septembre : 10,42 1 pour les zébus, 7,42 l pour les taurins et maximales en avril et mai $27,42 \mathrm{l}$ et $28,85 \mathrm{l}$ pour les zébus, $23,46 \mathrm{l}$ et $23,28 \mathrm{l}$ pour les taurins.

Les différences entre les maxima des deux groupes sont plus nettes que les différences entre les minima.

En étudiant les corrélations existant entre les besoins en eau et les caractéristiques climatiques, il est apparu que l’année pouvait être divisée en trois périodes :

- la promière, allant de fin juillet à la mioctobre, correspond à la saison des pluies ; la température est relativement faible $\left(29,6^{\circ} \mathrm{C} \pm\right.$ $104)$ le degré hygrométrique élevé $(61,4 \mathrm{p} .100 \pm$ $4,3)$ et l'épavoration faible $(17,2 \mathrm{~mm} \pm 1,1 \mathrm{~mm})$.
- la seconde, allant de la mi-octobre à la fin mars, correspond à la saison sèche ; la température est élevée $\left(31,2^{\circ} \mathrm{C} \pm 1,3^{\circ} \mathrm{C}\right)$, le degré hygrométrique faible $(16,3$ p. $100 \pm 3,4)$ l'évaporation forte $(69,1 \mathrm{~mm} \pm 7,6 \mathrm{~mm})$.

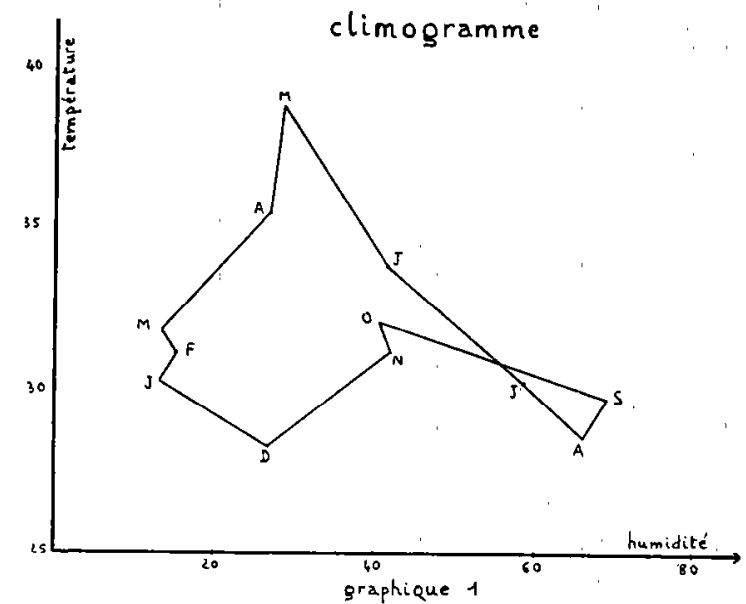

- la troisième, allant du début avril à la mijuillet, correspond au passage de la saison sèche à la saison des pluies. Elle se caractérise par une température très élevée $\left(36,9^{\circ} \mathrm{C} \pm 1,1^{\circ} \mathrm{C}\right)$ et surtout par une augmentation du degré hygrométrique $(31,9$ p. $100 \pm 5,3)$ dont la variabilité très grande dépend des précipitations orageuses. 
La consommation d'eau au cours des 3 périodes a été chez les zébus respectivement de $12,061 \pm 0,641 ; 21,711 \pm 1,511 ; 24,601 \pm$ 2,041 . Les différences entre les quantités bues au cours de 2 périodes consécutives sont significatives.

Pour les taurins, les quantités correspondantes ont été respectivement $9,181 \pm 1,801 ; 19,741 \pm$ 1,$291 ; 21,371 \pm 1,711$.

Les différences entre les quantités bues par les zébus et les taurins au cours de la même période sont significatives. Les zébus boivent toujours plus que les taurins.

\section{BESOINS EN EAU ET TENEUR EN EAU DES PLANTES}

Il serait illusoire étant donné les conditions de l'expérience de vouloir calculer des cœefficients de corrélation entre les besoins en eau des animaux et les quantités d'eau bues, cependant l'examen du schéma $\mathrm{n}^{\circ} 2$ dans lequel on a porté en abscisse les teneurs en eau des plantes et en ordonnée les quantités d'eau bues, permet de tirer quelques conclusions.

D'octobre à mars les quantités bues augmentent alors que le taux d'humidité des plantes diminue. En avril-mai, les variations du taux d'humidité n'ont plus d'action. En juin-juillet, il y a une nupture rapide, il semble que l'animal fasse son "plein d'eau ". Pendant l'hivernage les variations du taux d'humidité n'ont que peu d'importance.

L'action du climat est donc la seule cause de variation des besoins en eau à la fin de la saison sèche et pendant l'hivernage, alors qu'au début de la saison sèche la teneur en eau des fourrages a une influence certaine.

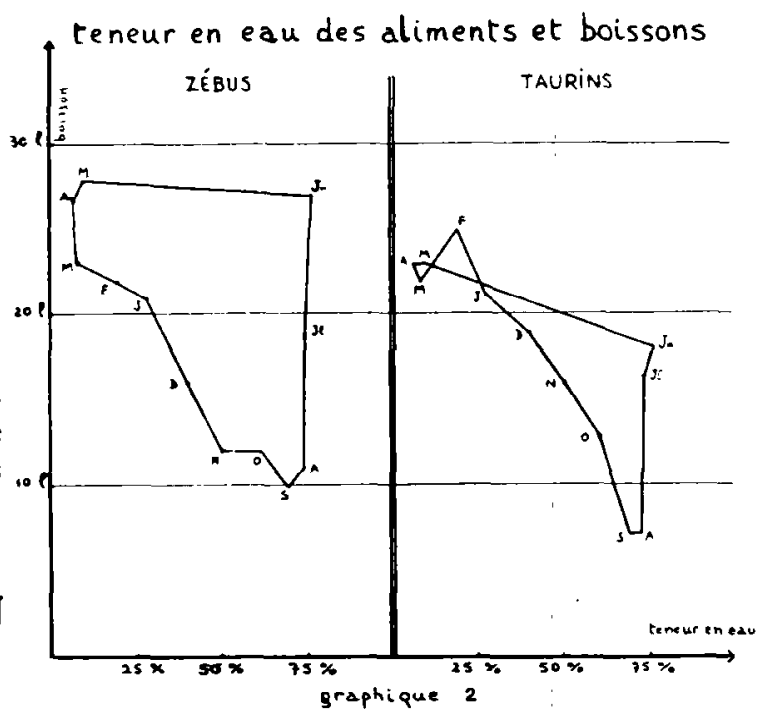

\section{CORRELATION ENTRE BESOINS EN EAU ET CLIMAT}

Nous avons calculé pour chacune des périodes définies précédemment les corrélations totales entre les besoins en eau et la température ambiante, le degré hygrométrique, l'évaporation. Puis, nous avons essayé de mesurer les actions propres de la température et du degré hygrométrique en calculant les coefficients de corrélation partielle entre les besoins en eau et l'une des caractéristiques du climat, l'autre restant constante.

\section{CORRELATION ENTRE LE BESOIN EN EAU ET LA TEMPERATURE}

Les cofficients de corrélation"entre les consornmations d'eau et la température au cours des trois périodes de l'année sont significatifs et positifs.

Tableau II

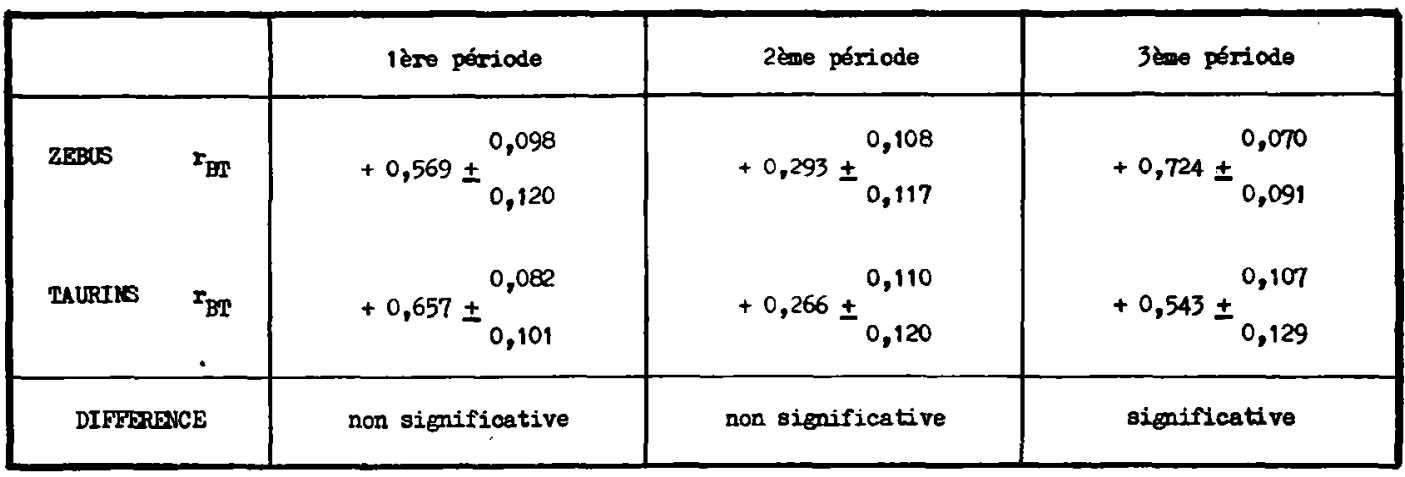


Pour un même groupe les différences entre les valeurs des coefficients pour deux périodes successives sont significalives.

Mais, pour une même période, ce n'est que pendant la $3^{\mathrm{e}}$ période que la différence entre les valeurs des coefficients des zébus et des taurins est significative, le cœfficient de corrélation des zébus étant le plus élevé.

\section{CORRELATION ENTRE LES BESOINS EN EAU ET LE DEGRE HYGROMETRI- QUE}

Les cœfficients de corrélation entre la consommation d'eau et le degré hygrométrique au cours des trois périodes de l'année sont significatifs et négatifs. (Tableau III).

Pour un même groupe, les différences entre les valeurs des coefficients pour deux périodes successives sont significatives.

Pour une même période les différences entre les cœfficients des zébus et ceux des taurins sont significatives pour les 3 périodes.

\section{CORRELATION ENTRE LES BESOINS EN EAU ET L'EVAPORATION}

Les cœfficients de corrélation entre les besoins en eau et l'évaporation au cours des 24 heures précédant l'observation sont significatifs et positifs. (Tableau IV).

Pour les zébus, les différences entre les valeurs des cofficients de deux périodes successives ne sont pas significatives, alors que pour les taurins elles le sont très nettement.

Pour chaque période les cœfficients des taurins sont significativement plus élevés que ceux des zébus.

L'évaporation au cours des 24 heures précédant l'abreuvement peut être considérée comme la résultante des actions conjuguées de la température, du degré hygrométrique, et du vent. La valeur des coefficients montre: que les taurins sont plus sensibles que les zébus aux actions du climat.

Tableau III

\begin{tabular}{|c|c|c|c|}
\hline & 1ère période & 2ème périade & 3ène période \\
\hline ZFiBus $\quad r_{\text {B.H. }}$ & $-0,635 \pm \frac{0,105}{0,086}$ & $-0,741 \pm \frac{0,062}{0,051}$ & $-0,830 \pm 0,061$ \\
\hline TAURINS $\quad r_{\text {B.H. }}$ & $-0,737 \pm 0,085$ & $-0,653 \pm 0,078$ & $-0,549 \pm 0,0,128$ \\
\hline DIFFERENCE & significative & significative & significative \\
\hline
\end{tabular}

Tableau IV

\begin{tabular}{|c|c|c|c|}
\hline & 1̇̀re période & 2ème période & 3ème période \\
\hline 7.EBUS $\quad r_{\text {B.E. }}$ & $+0,533 \pm 0,105$ & $+0,623 \pm 0,070$ & $+0,673 \pm \frac{0,080}{0,104}$ \\
\hline TAURINS $\mathbf{r}_{\mathrm{B}_{\bullet} \mathrm{E}_{\bullet}}$ & $+0,658 \pm 0,074$ & $+0,788 \pm 0,040$ & $+0,819 \pm 0,064$ \\
\hline IIFFERENCE & significative & significative & significative \\
\hline
\end{tabular}


Tableau V

\begin{tabular}{|c|c|c|c|}
\hline ZEBUS $\quad r_{\text {BT.H. }}$ & $\begin{array}{c}\text { lère période } \\
+0,319 \pm \begin{array}{r}0,137 \\
0,152\end{array}\end{array}$ & $\begin{array}{c}\text { 2ème période } \\
-0,269 \pm 0,118 \\
0,111\end{array}$ & $\begin{array}{l}\text { 3ème période } \\
+0,184 \pm \begin{array}{l}0,156 \\
0,167\end{array}\end{array}$ \\
\hline TAURINS $\mathbf{r}_{3 \mathrm{~A} . \mathrm{H} .}$ & $+0,421 \pm \frac{0,124}{0,142}$ & $-0,199 \pm \frac{0,121}{0,115}$ & $-0,229 \pm 0,165$ \\
\hline DIFFERPACE & non significative & non significative & non significative \\
\hline${ }^{S_{B E} . T} \mathrm{~T}$ & $-0,457 \pm \begin{array}{l}0,119 \\
0,147\end{array}$ & $-c, 737 \pm 0,072$ & $-0,608 \pm \begin{array}{l}0,118 \\
0,095\end{array}$ \\
\hline TAURINS $r_{B H . T}$ & $-0,582 \pm 0,116$ & $-0,638 \pm{ }_{C, 068}^{0,079}$ & $-0,208 \pm \begin{array}{l}0,166 \\
0,154\end{array}$ \\
\hline DIFFERDXCE & significative & sigificative & significative \\
\hline
\end{tabular}

ACTION COMBINEE DE LA TEMPERATURE ET DU DEGRE HYGROMETRIQUE SUR LES BESOINS EN EAU

$\mathrm{Si}$ le calcul des coefficients des corrélations entre les besoins en eau et l'évaporation permet d'avoir une idée des actions globales du climat, le calcul des coefficients de corrélation partielle entre les besoins en eau et une caractéristique du climat, l'autre restant constante, permet de mesurer avec plus d'exactitude l'influence réelle de chacun des facteurs climatiques, lorsque ces facteurs varient entre certaines limites. (Tabl. V).

CGFFICIENTS DE CORRELATION ENTRE LES BESOINS EN EAU ET LA TEMPERATURE AMBIANTE, le degré hygrométrique étant constant.

Pour un même groupe les différences entre les coefficients de deux périodes successives sont significatives.

Pendant la première période - saison des pluies - (température basse, degré hygrométrique élevé), la corrélation partielle entre les besoins en eau et la température à degré hygrométrique constant (rBT.H) est positive pour les deux groupes et bien que le coefficient des taurins $(+0,421)$ soit plus élevé que celui des zébus $(+0,319)$ la différence n'est pas signifcative.

Pendant la $2^{\mathrm{e}}$ période - saison sèche (température moyenne, degré hygrométrique faible), le coefficient de corrélation partielle est négatif. Il a pour les deux groupes, une valeur très faible, presque à la limite de signification. La différence entre les coefficients des zébus $(-0,269)$ et des taurins $(-0,199)$ n'est pas significative.

Pendant la $3^{e}$ période - fin saison sèche, début saison des pluies - (température élevée, fort degré hygrométrique), le coefficient de corrélation partielle est positif et significatif pour lcs taurins alors que chez les zébus, s'il est positif, il est très faible et à peine significativement différent de zéro.

En conclusion pendant la saison des pluies et la saison sèche les réactions des zébus et des taurins aux variations de température à degré hygrométrique constant sont très comparables. Ce n'est que lorsque la température s'élève, le degré hygrométrique étant lui-même élevé, que le comportement diffère. Les taurins semblent avoir atteint un seuil thermique au delà duquel les besoins en eau n'augmentent presque plus. 

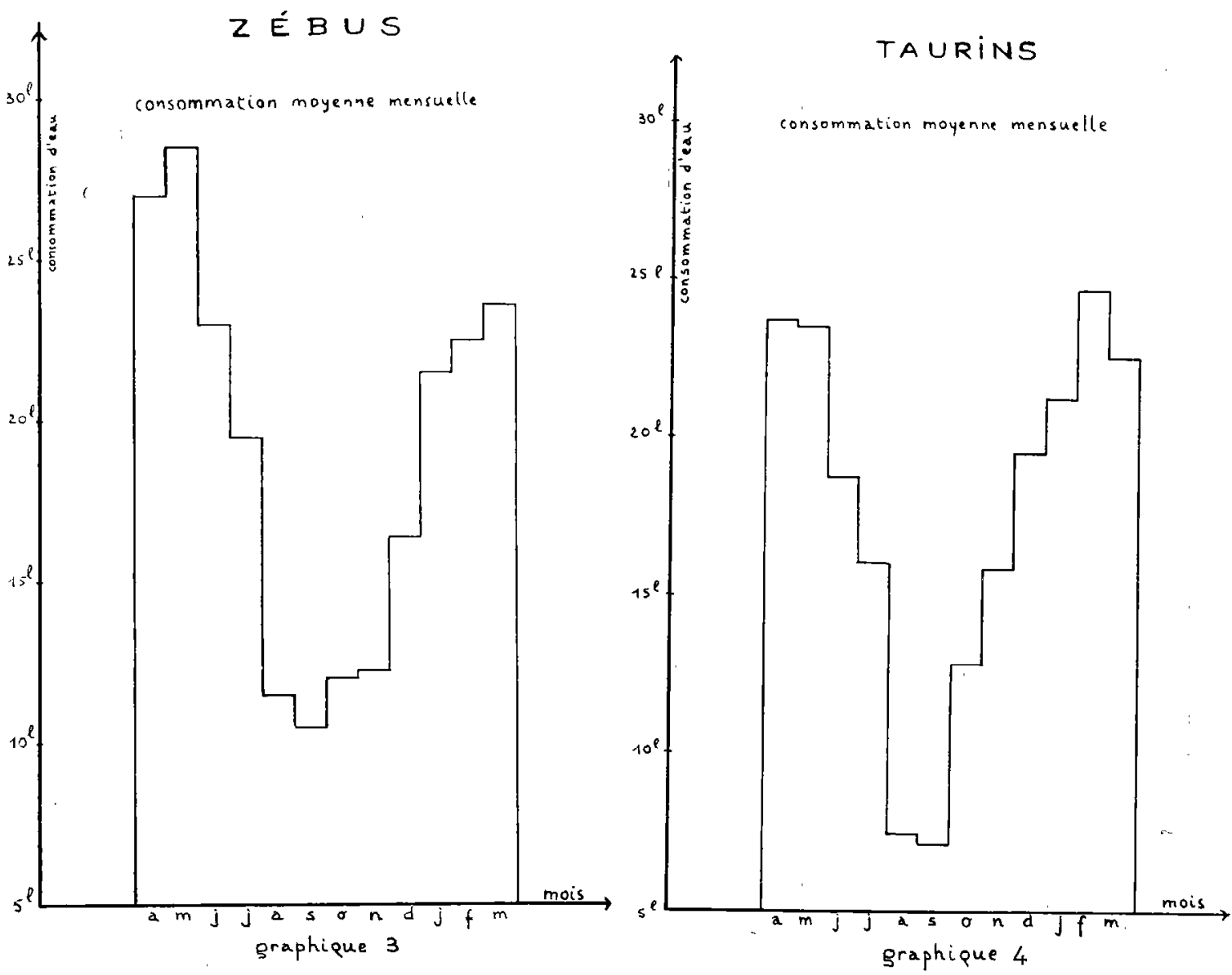

COFFICIENTS DE CORRELATION ENTRE LES BESOINS EN EAU ET LE DEGRE HYGROMETRIQUE, la température étant constante.

Pour les zébus, les différences entre les valeurs des coefficients de deux périodes successives sont significatives, alors que, pour les taurins, seule la différence entre les coefficients des $2^{\mathrm{e}}$ et $3^{\mathrm{e}}$ périodes est significative.

Pendant la première période, la corrélation entre les besoins en eau et le degré hygrométrique, la température étant constante, est négative et la différence entre le cœfficient des taurins $(-0,582)$ et celui des zébus $(-0,457)$ est significative.

Pendant la $2^{\mathrm{e}}$ période les coefficients de corrélation partielle sont négatifs et la différence entre les coefficients des zébus $(-0,737)$ et des taurins $(-0,638)$ est significative.
Enfin pendant la $3^{\mathrm{e}}$ période, les coefficients de corrélation partielle sont négatifs et significatif́s pour les deux groupes et la corrélation est significativement plus élevée chez les zébus $(-0,608)$, que chez les taurins $(-0,208)$.

\section{CONCLUSION}

En combinant les résultats obtenus on peut donc dire quau cours de la première période de l'année qui correspond à l'hivernage, lorsque la température est basse, et le degré hygrométrique élevé, dans les deux groupes, à une élévation de température correspond une augmentation de la consommation d'eau alors qu'un accroissement du degré hygrométrique a une action inverse.

Si les zébus et les taurins réagissent de façon très voisine à l'action de la température, les taurins réagissent d'une façon plus nette aux variations du degré hygrométrique. 
Au cours de la seconde période de l'année qui correspond à la saison sèche, lorsque la température a une valeur moyenne et que le degré hygrométrique est faible, laction des variations de température est très faible et identique dans les deux groupes, alors que l'action des variations du degré hygrométrique est très nette et plus élevée chez les zébus que chez les taurins.

Au cours de la troisième période qui correspond à la fois à la saison sèche et au début de l'hivernage, les zébus et les taurins ont des comportements différents. Les variations de température ont une action très faible chez les taurins, négligeable chez les zébus.

La corrélation entre les besoins en eau et les variations $\mathrm{du}$ degré hygrométrique est plus élevée chez les zébus que chez les taurins.

En résumé, les besoins en eau étant en fait une mesure de l'action des mécanismes thermorégulateurs, il semble que, chez les taurins comme chez les zébus, ce soit surtout la valeur du degré hygrométrique qui intervienne dans leur régulation, et lorsque la température atteint des valeurs voisines de la température corporelle, l'action du degré hygrométrique est bien plus forte sur les zébus que sur les taurins.

Compte tenu de la valeur des différents coefficients de corrélation partielle, les taurins supportent mieux les hauts degrés hygrométriques que les zébus. Cette conclusion n infirme pas les constatations faites sur le terrain. Les zébus vivent surtout dans la zone sahélo-soudanienne, très sèche neuf mois sur douze, et les taurins dans la zone Guinéenne, humide huit mois sur douze.

Des observations sont en cours pour préciser nos conclusions. Dans un prochain article nous exposerons les résultats obtenus dans l'étude de l'influence du climat sur la température corporelle, le rythme respiratoire et le pouls des zébus, des taurins et de leurs métis.

Nous remercions M. Vessereau, professeur à l'Institut de statistique de l'Université de Paris, qui a vérifié et confirmé notre analyse statistique.

Centre de recherches zootechniques de l'Afrique accidentale francaise. Bamako Soudan.

\section{BIBLIOGRAPHIE}

1. BRODY et coll. - Environmental Physiology with Special Reference to Domestic Animals Missouri Agric. Stat. Columbia, 39 numéros de Res. Bull. Mo. Agric. Exp. Sta., pp. 423-608.

2. Newburgh (L.-H.) et Jonston (M.-W.). The Insensible Loss of Water. Physiol. Rev., 1942, 22, 1-18.

3. Osborne (W.-A.). - Some New Aspects of the Function of the Skin in the Temperature Regulation. J. Physiol., 1922, 57, XXVI Proc.

4. Pacot (J.). - Température corporelle des zébus et métis zébus-taurins sous les tropiques. Rev. Elev. Médec. vét. Pays trop., 1956,9 (1), $21-42$.

5. Pinson (E.-A.). - Evaporation from Human Skin with Sweat Glands Inactived. Amer. J. Physiol., 1942, 137, 492-503.

6. Seath (D.-M.) and Miller (G.-D.). - Effect of Shade and Sprinkling with Water on Summer Comfort of Jersey Cows. J. Dairy Sci., 1947, 30, 255-61.

7. Simonnet $(\mathrm{H}$.$) . - L'eau. Besoins de l'orga-$ nisme, métabolisme, influence de l'abreuvement sur la production animale. Rev. Elev. Méd. vét. Pays trop., 1955, 8 (2-3), 179-208.

8. Whitehouse Aar, Hancolk (W.) et Haldane (J.-S.). - The Osmotic Passage of Water and Gass Through the Human Skin. Proc. Roy. Soc. London B., 1932, 3, 412-29.

9. Harker (K.-W.), Taylor (J.-I.) et Rollinson (D.-H.-L.) - Studies on the Habits of Zebu Cattle; I. Preliminary Observations on Grazing Habits. J. Agric. Sci., 1955, 45, 193-8.

10. TAYlor (J.-I.), Rollinson (D.-H.-L.) et HARKER (K.-W.). - Studies on the Habits of Zebu Cattle ; II. Individual and Group Variation within a Herd. J. Agric. Sci., $1955,45,257-63$.

11. Rollinson (D.-H.-L.), HarKer (K.W.) et TAY'LOR (J.-I.). - Studies on the Habits of Zebu Cattle ; III. Water Consumption of Zebu Cattle. J. Agric. Sci., 1955, 46, 123-9. 


\section{SUMMARY}

\section{The water requirement of european and zebu cattle in Tropical Africa \\ (French Sudan - French West Africa).}

The authors have studied the water requirement of zebu and european cattle maintained under range conditions in the Sudanese Zone. During the rainy season zebu and european cattle drink an average of 12.06 litres and 9.18 litres daily respectively; during the dry season the quantities are 21.71 litres and 15.74 litres; during the transitional seasons, april-may averages arc 28.85 and 23.46 litres, and during September 10.42 and 7.42 litres.

The degree of humidity of the forage influences greatly the quantity of water consumed. The hygrometric reading has a greater influence than the temperature particularly in the zebus. Correlation between water needs and evaporation during 24 hours periods is a good measurement of the total action of climate, and european cattle are more markedly affected by the climate than the zebus

\section{RESUMEN}

\section{Necesidades en agua de los taurins y de los cebues en la zona tropical (Sudàn, Africa Occidental Francesa).}

Los autores estudian las necesidades en agua de los cebúes y taurins en cría extensiva en la zona del Sudán y la influencia del clima sobre éstas necesidades. En la estación lluviosa los cebúes beben en promedio 12,06 litros de agua por dia y los taurins 9,18 litros; en la estación seca las cantidades respectivas son 21,71 y 15,74 litros. Durante los períodos de transicíon, en Abril y Mayo, los cebúes consumen en promedio 28,85 litros y los taurins 23,46 litros y en septiembre, respectivamente 10,42 y 7,42 litros.

El tenor acuso de los forrajes tiene una acción definida sobre la cantitad de agua bebida ; el grado higrométrico tiene una mayor influencia que la temperatura sobretodo en los cebúes. La correlación entre las necesidades en agua y la evaporación en el curso de las 24 horas precedentes a la observación, dá una excelente medida sobre la accíon global del clima, al cual son más sensibles los taurins gue los cebúes. 